\title{
Bioecologia do vetor Diaphorina citri e transmissão de bactérias associadas ao huanglongbing
}

\author{
José Roberto Postali Parra ${ }^{1}$, João Roberto Spotti Lopes ${ }^{1 *}$, \\ Mariuxi Lorena Gómez Torres ${ }^{1}$, Dori Edson Nava ${ }^{2} \&$ Paulo Eduardo Branco Paiva ${ }^{1}$
}

\section{RESUMO}

Com a descoberta do huanglongbing (HLB, ex-greening) no Brasil, em 2004, o psilídeoasiático-dos-citros, Diaphorina citri Kuwayama (Hemiptera: Psyllidae), assumiu o status de praga-chave na citricultura pela sua capacidade de transmitir bactérias do gênero Candidatus Liberibacter spp., associadas a esta enfermidade. Por ser uma espécie exótica e de importância recente, há poucas informações sobre a biologia e ecologia desse psilídeo nas condições do Brasil, as quais são necessárias para o seu manejo. Portanto, este artigo busca compilar dados disponíveis sobre danos, dinâmica populacional e biologia de $D$. citri, com ênfase na influência de plantas hospedeiras, temperatura e umidade relativa do ar no seu desenvolvimento (duração e viabilidade dos estágios de ovo e ninfal), razão sexual e fecundidade. São descritos métodos alternativos de controle $D$. citri, com destaque para o controle biológico e a utilização do ectoparasitoide Tamarixia radiata (Waterston). Com base nas exigências térmicas deste parasitoide e de $D$. citri, elaborou-se o zoneamento ecológico para prever o número de gerações anuais de ambas as espécies no estado de São Paulo. Finalmente, são discutidos os mecanismos de transmissão de $C a$. Liberibacter spp. por D. citri, baseando-se nas informações disponíveis sobre a interação patógeno-vetor.

Termos de indexação: greening dos citros, inseto vetor, biologia, controle biológico, mecanismos de transmissão.

\section{SUMMARY \\ Biology and ecology of the vector Diaphorina citri and transmission of bacteria associated with huanglongbing}

The citrus disease huanglongbing (HLB, or greening) was detected in Brazil in 2004. The already established Asian citrus psyllid, Diaphorina citri Kuwayama (Hemiptera: Psyllidae) became an important pest of citrus after the emergence of HLB due to its ability to transmit bacterial species of the genus Candidatus Liberibacter spp., which is associated with HLB. Although D. citri was already established in Brazil, limited information about its biology and ecology is available. The goal of this article is to review available data on the biology and population dynamics of D. citri. Emphasis will be given to the role of host plant, temperature and relative humidity on its development (duration and viability of egg and nymphal phases), sex ratio and fecundity. We also discuss approaches to control $D$. citri, including biological control and the impact of an

\footnotetext{
${ }^{1}$ Esalq/USP, Av. Pádua Dias, 11, Caixa Postal 9, 13418-900 Piracicaba/SP

*Autor para correspondência - E-mail: jlopes@esalq.usp.br

${ }^{2}$ Embrapa Clima Temperado, Pelotas/RS
} 
ectoparasitoid, Tamarixia radiata (Waterson), on psyllid populations. In addition, we present a model forecasting the number of $D$. citri and T. radiata generations per year in Sao Paulo state based on thermal requirements of these pests. Lastly, we discuss the transmission mechanisms of $C a$. Liberibacter spp. by D. citri.

Index terms: citrus greening, insect vector, biology, biological control, transmission mechanisms.

\section{INTRODUÇÃO}

O psilídeo-asiático-dos-citros Diaphorina citri Kuwayama, é referido no Brasil desde 1942 (Costa Lima, 1942); entretanto, a ocorrência de bactérias do gênero Candidatus Liberibacter spp., associadas ao huanglongbing (HLB, ex-greening), só foi constatada, no Brasil, em 2004, com a observação de plantas com sintomas típicos desta doença no estado de São Paulo (Coletta-Filho et al., 2004; Teixeira et al., 2005). Trata-se, portanto, de um caso peculiar, pois na maioria das vezes, como na Flórida (EUA), poucos anos após o registro deste inseto vetor, que ocorreu em 1998, constatou-se a disseminação do HLB (Figura 1).

É uma questão a ser elucidada, pois mesmo com a presença constante do psilídeo em pomares de São Paulo e de outros estados brasileiros, durante todos estes anos, o HLB só foi constatado 62 anos após a referência da ocorrência da praga. Diante desta questão, todos os aspectos biológicos estudados (e não são muitos) devem ser revistos, pois pode tratar-se de um novo strain ou linhagem de D. citri com características diferentes daquelas populações ocorrentes desde 1942. Deve-se salientar, no entanto, que outros fatores podem ter condicionado a disseminação relativamente recente de HLB, como a possível introdução de materiais propagativos de citros ou mesmo de outras rutáceas exóticas infectados com o patógeno. A descoberta da nova espécie $C a$. Liberibacter americanus no Brasil é uma evidência de que o patógeno possa ter sido introduzido via outras rutáceas, uma vez que esta espécie bacteriana não havia sido registrada em citros até então em outras regiões onde a doença é endêmica. O psilídeo Trioza erytreae (Del Guercio), outra espécie vetora de $\mathrm{Ca}$. Liberibacter spp. originária da região etiópica e confinada a regiões altas da África Tropical (Bové, 2006), não é referida no Brasil.

Assim, o objetivo deste trabalho é expor os dados obtidos com $D$. citri a partir da referência da doença entre nós, com ênfase à sua bioecologia, dinâmica populacional e distribuição espacial e temporal, inimigos naturais como opção de controle e mecanismos de transmissão da bactéria.

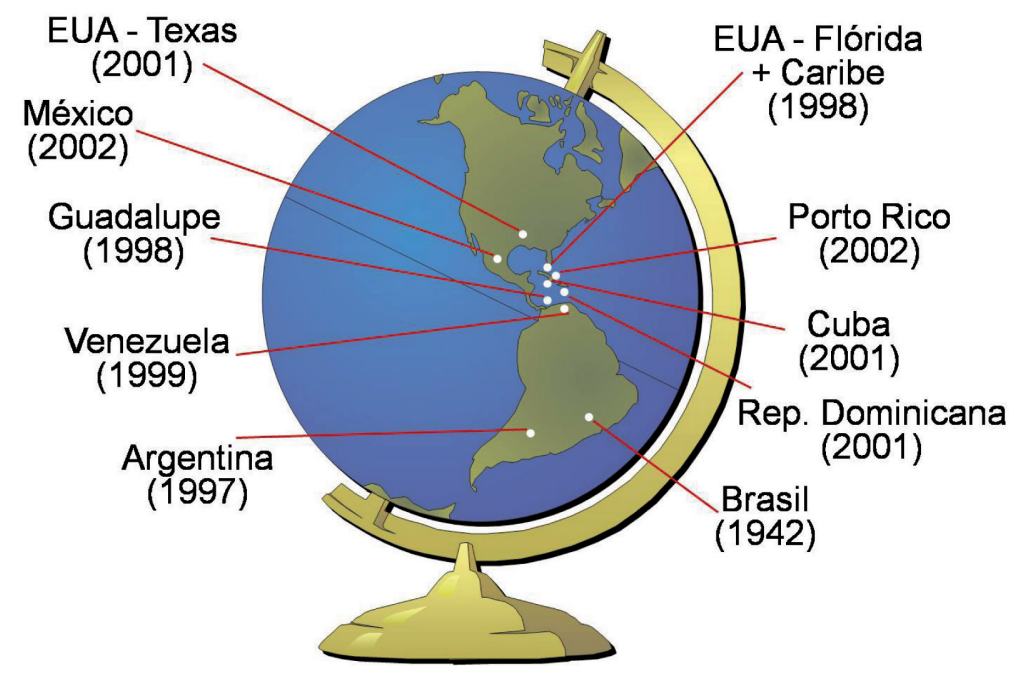

Figura 1. Registro de Diaphorina citri em diferentes países (Halbert \& Nuñez, 2004; Costa Lima, 1942). 


\section{IMPORTÂNCIA DO PSILÍDEO}

Este inseto foi por muitos anos considerado uma praga secundária de citros no Brasil (Gallo et al., 2002), até o registro do HLB em 2004, quando passou a ser a mais importante praga da cultura, como tem sido em todas as regiões citrícolas em que ocorre no mundo. Trata-se de um inseto cosmopolita, originário da Ásia e que, atualmente, possui ampla distribuição geográfica, nos continentes asiático (Bové, 2006) e americano (Halbert \& Nuñez, 2004).

Em condições de temperatura e umidade relativa que favorecem o seu crescimento populacional, este inseto pode causar danos diretos problemáticos, tais como deformações de folhas jovens, seca de brotações e presença de fumagina (Halbert \& Manjunath, 2004; Michaud, 2004). Tais danos são resultantes de injeção de toxinas pelas ninfas que levam a alterações no crescimento das partes atacadas. As folhas deformam-se pela retirada da seiva elaborada de plantas hospedeiras. A fumagina, resultante do excesso de honeydew, pode levar a secamento das estruturas vegetais atacadas. Tudo dependerá do nível populacional e, em altas densidades, pode haver abscisão de folhas e mesmo de todo o ramo infectado (Hoy \& Nguyen, 2001; Paiva, 2009). A despeito desses danos verificados sob altas populações, Michaud (2004) observou deformações foliares irreversíveis quando uma única ninfa se alimentou por 24 horas. Os adultos de D. citri estão, como as ninfas, mais frequentemente associados a ramos novos (brotações), porém podem se alimentar de folhas maduras. Ramos em crescimento têm sido o principal fator determinante da ocorrência e do aumento populacional de D. citri (Yamamoto et al., 2001; Tsai et al., 2002; Paiva, 2009).

Embora cause danos diretos em citros, $D$. citri tornou-se a principal praga da cultura pela sua capacidade de transmitir as bactérias associadas ao HLB, $C a$. Liberibacter americanus, $C a$. L. asiaticus e $\mathrm{Ca}$. L. africanus. Atualmente, cerca de $5 \%$ das plantas cítricas do estado de São Paulo encontram-se com sintomas de HLB. Avaliações qualitativas preliminares dos danos devido à doença foram registradas por Bassanezi et al. (2006) que observaram reduções de $70 \%$ na produção de frutos em plantas com 4 a 6 anos de idade com mais de $60 \%$ da copa com sintomas de
HLB. Segundo os autores, a redução na produção de frutos foi diretamente proporcional à área da planta com sintomas. As plantas atacadas morrem e se não forem tomadas medidas, o problema poderá se alastrar e dizimar grandes pomares, como registrado em diversos países do mundo.

\section{BIOECOLOGIA DE D. citri}

O psilídeo D. citri ocorre preferencialmente em ramos novos de plantas da família Rutaceae; são referidas cerca de 21 espécies de hospedeiros, embora em algumas delas não ocorra desenvolvimento completo e, em outras, o inseto coloque apenas ovos, sem sequência de crescimento na referida planta. A espécie Murraya paniculata (L.) Jack, conhecida popularmente como murta-de-cheiro, utilizada como cerca viva ou ornamental no Brasil, é um dos principais hospedeiros deste psilídeo.

Os ovos de coloração amarela são depositados nas gemas recém brotadas e as ninfas desenvolvem-se de forma sincronizada com o crescimento dos ramos. Os adultos são mais frequentemente associados a ramos em crescimento, podendo se alimentar de folhas maduras (Figura 2). A disponibilidade de ramos novos é o fator determinante para ocorrência e incremento populacional de D. citri.

Estudos realizados no Brasil por Nava et al. (2007) demonstraram que o desenvolvimento ovo-adulto de $D$. citri em limão Cravo (Citrus limonia Osbeck), murta-decheiro (M. paniculata) e tangerina Sunki (C. sunki hort. ex Tanaka), não foi afetado com relação à duração, sendo próxima a 18 dias nos três hospedeiros (Tabela 1). A razão sexual também não foi influenciada pelo hospedeiro, sendo

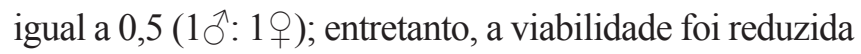
quando o inseto se desenvolveu em tangerina Sunki, pois a mortalidade ninfal foi de 44,6\% (Tabela 1), mostrando ser este um hospedeiro menos adequado à praga. Quanto à fecundidade, observou-se que o maior número de ovos foi colocado em plântulas de murta-de-cheiro (348,4 ovos) e limão Cravo (265,1 ovos), diferindo significativamente da tangerina Sunki (166,2 ovos) (Figura 3). Tais valores são inferiores aos relatados por Liu \& Tsai (2000) quando utilizaram murta-de-cheiro como hospedeiro (626 ovos) a $26^{\circ} \mathrm{C}$. Estas diferenças podem estar associadas às condições de estudo, strains do psilídeo ou mesmo de gerações de laboratório. 


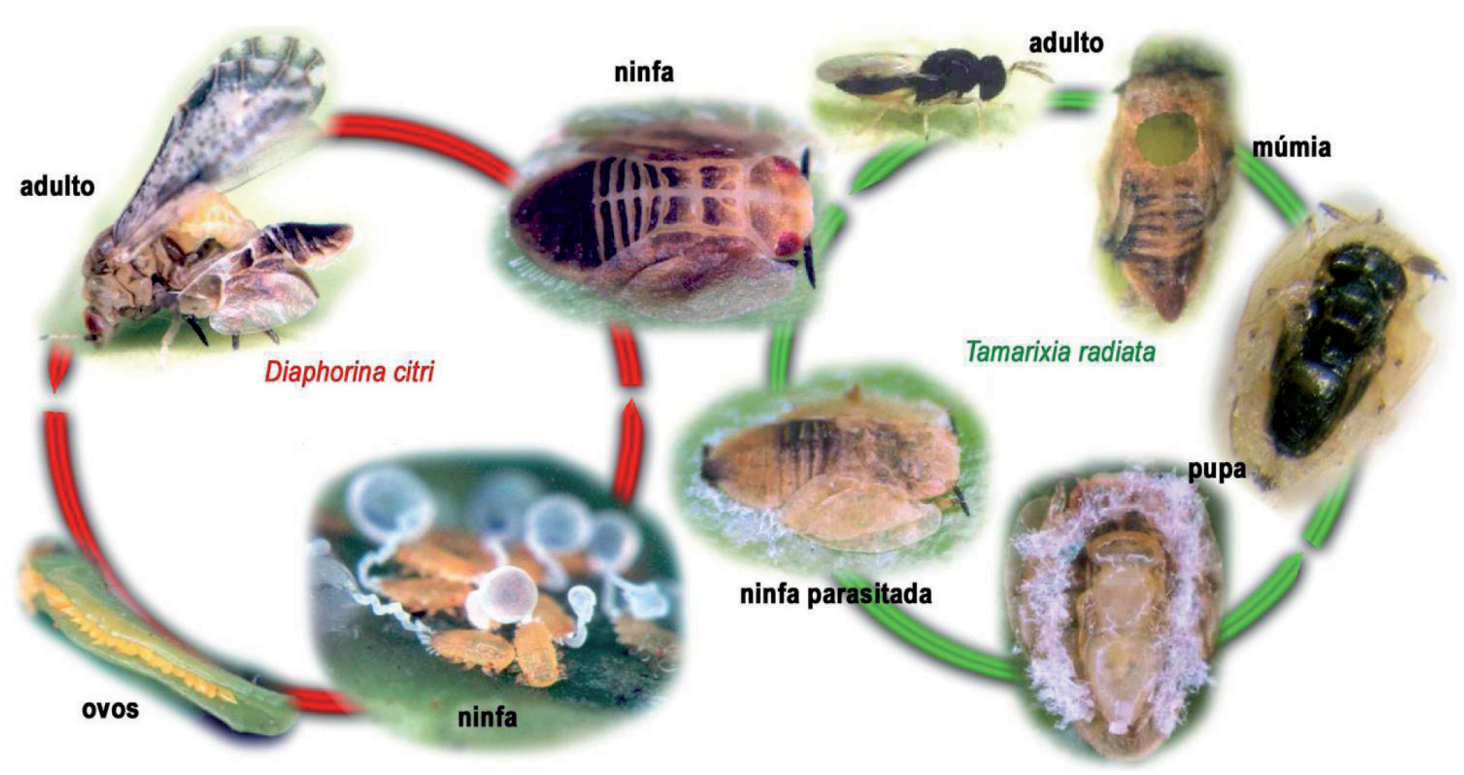

Figura 2. Ciclo biológico de Diaphorina citri e Tamarixia radiata (Gómez Torres, 2009).

Tabela 1. Duração e sobrevivência das fases de ovo e ninfa de Diaphorina citri criado em diferentes hospedeiros. Temperatura de $24 \pm 2^{\circ} \mathrm{C}$, UR de $70 \pm 10$ e fotofase de 14 horas

\begin{tabular}{|c|c|c|c|c|c|}
\hline \multirow{2}{*}{ Hospedeiro } & \multicolumn{2}{|c|}{ Duração (dias) } & \multicolumn{2}{|c|}{ Viabilidade } & \multirow{2}{*}{$\begin{array}{c}\text { Razão sexual ns } \\
\left(q / q+{ }^{\lambda}\right)\end{array}$} \\
\hline & Ovo ${ }^{\text {ns }}$ & Ninfa & Ovo ns & Ninfa & \\
\hline Limão Cravo & $3,61 \pm 0,62$ & $14,00 \pm 056 \mathrm{a}$ & $87,50 \pm 2,49$ & $82,35 \pm 6,27 \mathrm{a}$ & $0,50 \pm 0,05$ \\
\hline Murta-de-cheiro & $3,63 \pm 0,49$ & $14,11 \pm 0,28 \mathrm{a}$ & $88,36 \pm 1,93$ & $88,82 \pm 5,21 \mathrm{a}$ & $0,50 \pm 0,06$ \\
\hline Tangerina Sunki & $3,57 \pm 0,64$ & $13,46 \pm 0,51 \mathrm{a}$ & $89,78 \pm 2,35$ & $44,61 \pm 9,10 \mathrm{~b}$ & $0,47 \pm 0,07$ \\
\hline
\end{tabular}

Médias seguidas da mesma letra, na coluna, não diferem entre si, pelo teste de Tukey $(\mathrm{P} \pm 0,05)$. ns = não significativo.
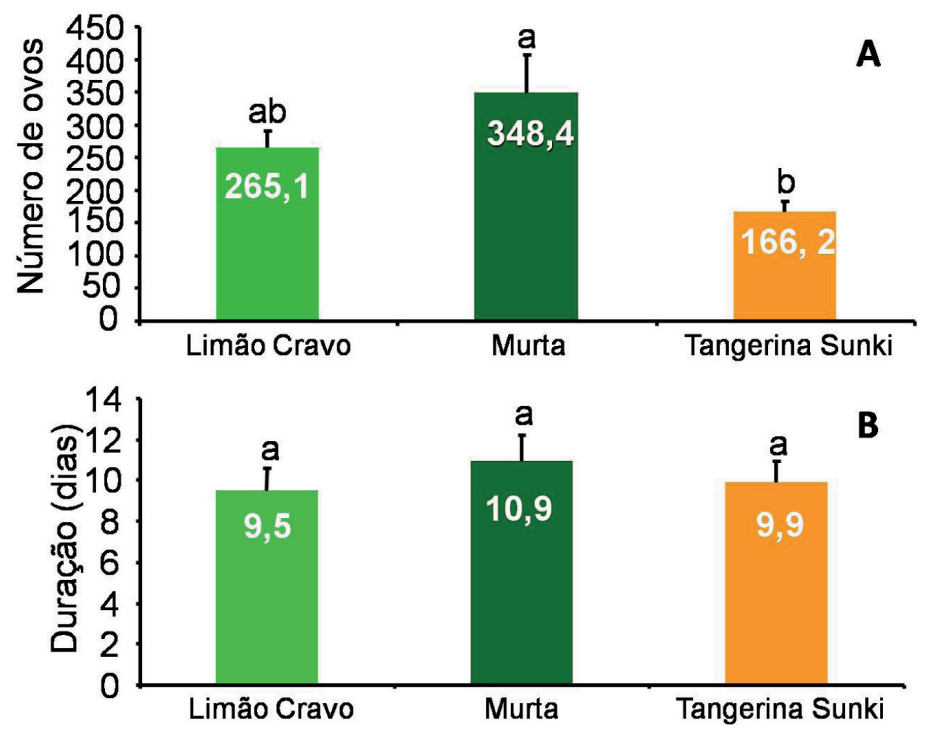

Figura 3. Número médio de ovos (A) e período de pré-oviposição (B) de Diaphorina citri criado em diferentes hospedeiros. Temperatura de $24 \pm 2^{\circ} \mathrm{C}$, UR de $70 \pm 10 \%$ e fotofase de 14 horas. Médias seguidas da mesma letra, não diferem entre si, pelo teste de Tukey $(\mathrm{P} \pm 0,05)$ (Nava et al. 2007). 
Tabela 2. Médias ( \pm DPM) da duração dos ínstares de Diaphorina citri, criado em diferentes hospedeiros. Temperatura de $24 \pm 2^{\circ} \mathrm{C}$, UR de $70 \pm 10 \%$ e fotofase de 14 horas

\begin{tabular}{|c|c|c|c|c|c|c|}
\hline \multirow{2}{*}{ Hospedeiro } & \multicolumn{6}{|c|}{ Duração } \\
\hline & $1^{\circ}$ Ínstar & $2^{\circ}$ Ínstar & $3^{\circ}$ Ínstar & $4^{\circ}$ Ínstar & $5^{\circ}$ Ínstar & Total \\
\hline Limão Cravo & $\begin{array}{c}2,57 \pm 0,14 \mathrm{a} \\
(2-3)\end{array}$ & $\begin{array}{c}2,21 \pm 0,11 \mathrm{a} \\
(2-3)\end{array}$ & $\begin{array}{c}1,79 \pm 0,15 \text { a } \\
(1-3)\end{array}$ & $\begin{array}{c}2,43 \pm 0,23 \mathrm{a} \\
(1-4)\end{array}$ & $\begin{array}{c}5,57 \pm 0,29 b \\
(4-7)\end{array}$ & 14,57 \\
\hline Murta-de-cheiro & $\begin{array}{c}2,43 \pm 0,17 \mathrm{a} \\
(2-4)\end{array}$ & $\begin{array}{c}2,12 \pm 0,18 \mathrm{a} \\
(1-3)\end{array}$ & $\begin{array}{c}2,71 \pm 0,22 \mathrm{a} \\
(1-4)\end{array}$ & $\begin{array}{c}3,07 \pm 0,20 \mathrm{ab} \\
\quad(2-4)\end{array}$ & $\begin{array}{c}4,57 \pm 0,20 \mathrm{~b} \\
(4-6)\end{array}$ & 14,93 \\
\hline Tangerina Sunki & $\begin{array}{c}2,38 \pm 0,15 \mathrm{a} \\
(2-4)\end{array}$ & $\begin{array}{c}2,08 \pm 0,14 \mathrm{a} \\
(1-3)\end{array}$ & $\begin{array}{c}2,00 \pm 0,16 \mathrm{a} \\
(1-3)\end{array}$ & $\begin{array}{c}2,69 \pm 0,17 \mathrm{a} \\
(2-4)\end{array}$ & $\begin{array}{c}5,00 \pm 0,16 \mathrm{~b} \\
(4-6)\end{array}$ & 14,15 \\
\hline
\end{tabular}

Médias seguidas da mesma letra, na coluna, não diferem entre si, pelo teste de Tukey $(\mathrm{P} \leq 0,05)$.

O período de pré-oviposição foi semelhante nos três hospedeiros tratamentos, sendo próximo a 10 dias e as longevidades das fêmeaas foram maiores que as dos machos, independente da planta hospedeira em que se criaram (Nava et al., 2007). A postura, no entanto, é concentrada nos primeiros 10 dias e as ninfas apresentam cinco ínstares, independente do hospedeiro estudado, sendo o último, o mais longo, com cerca de cinco dias de duração (Tabela 2) (Nava et al., 2007).

\section{EFEITO DA TEMPERATURA E DA UMIDADE RELATIVA NO DESENVOLVIMENTO DE D. citri}

No Brasil, a influência da temperatura no desenvolvimento de $D$. citri foi estudada por Nava et al. (2007). Em limão Cravo, a duração média do período embrionário variou de 7,7 dias a $18^{\circ} \mathrm{C}$, a 2,6 dias a $32^{\circ} \mathrm{C}$ (Tabela 3). A faixa térmica estudada não afetou a viabilidade dos ovos que oscilou entre 81,6 e $95,2 \%$ (Tabela 4). Estes valores estão próximos aos relatados nos EUA por Liu \& Tsai (2000), que determinaram, em murta-de-cheiro, uma duração de 4,1 dias e uma viabilidade de $95,5 \%$ a $25^{\circ} \mathrm{C}$.

O limiar térmico inferior de desenvolvimento (Tb) da fase de ovo foi de $12^{\circ} \mathrm{C}$ e a constante térmica $(\mathrm{K})$ de 52,6 GD (Tabela 5) (Nava et al., 2007). Liu \& Tsai (2000) determinaram uma $\mathrm{Tb}$ de $8,9^{\circ} \mathrm{C}$ e uma constante térmica de 67,6 GD, para $D$. citri criada em murta-decheiro nos EUA, havendo, portanto, uma diferença próxima a $3^{\circ} \mathrm{C}$ no valor da $\mathrm{Tb}$ entre as populações de origem brasileira e norte-americana.
Para a fase de ninfa, houve uma variação de 9,4 a 35,8 dias, na faixa térmica de 32 a $18^{\circ} \mathrm{C}$ (Tabela 3 ), sendo que, entre 25 e $30^{\circ} \mathrm{C}$, a duração não diferiu entre as temperaturas estudadas. A viabilidade foi próxima de $70 \%$ na faixa térmica estudada, com exceção da temperatura de $32^{\circ} \mathrm{C}$, que proporcionou $15 \%$ de viabilidade das ninfas (Tabela 4). Para esta fase, a Tb e a $\mathrm{K}$ foram de $13,9^{\circ} \mathrm{C}$ e $156,88 \mathrm{GD}$, respectivamente (Nava et al. 2007), sendo superiores aos valores determinados por Liu \& Tsai (2000).

Para o ciclo biológico (ovo-adulto), houve uma variação de 43,5 a 12,1 dias na faixa de 18 a $32^{\circ} \mathrm{C}$ (Tabela 3). A $25^{\circ} \mathrm{C}$ determinou-se uma duração de 17,1 dias (Nava et. al. 2007), valor próximo aos 16,9 dias relatados por Liu \& Tsai (2000). A viabilidade desse período variou de 69,9 a $64,1 \%$ na faixa de 18 a $30^{\circ} \mathrm{C}$, sendo, contudo, de $12,2 \%$ a $32^{\circ} \mathrm{C}$ (Nava et. al. 2007). Embora na natureza, o inseto deva procurar microclimas favoráveis, a exposição a temperaturas elevadas por tempo prolongado deve afetar o seu desenvolvimento, e, portanto, não devem ser recomendadas para criação do inseto em laboratório (Tabela 4). Resultado semelhante $(75,4 \%)$ foi registrado por Liu \& Tsai (2000), quando D. citri foi criada em murta-de-cheiro a $25^{\circ} \mathrm{C}$, na Flórida, EUA. $\mathrm{A} \mathrm{Tb}$ e a $\mathrm{K}$ do período ovo-adulto foram de $13,5^{\circ} \mathrm{C}$ e 210,9 GD. As variações encontradas entre os estudos de Liu \& Tsai (2000) e Nava et al. (2007) podem ser devidas às diferentes populações de $D$. citri estudadas.

Observações realizadas durante três gerações de D. citri em campo na região de Piracicaba, SP, evidenciaram que a fase de ovo foi a que apresentou as maiores mortalidades, com 61,70 e $65 \%$ na $1^{\text {a }}$, 
Tabela 3. Duração média ( \pm DPM) (dias) das fases de ovo e ninfa e do ciclo biológico (ovo-adulto) de Diaphorina citri criado em limão Cravo em diferentes temperaturas. UR de $70 \pm 10 \%$ e fotofase de 14 horas

\begin{tabular}{cccc}
\hline \multirow{2}{*}{ Temperatura $\left({ }^{\circ} \mathrm{C}\right)$} & \multicolumn{3}{c}{ Duração (dias) } \\
\cline { 2 - 4 } & Ovo & Ninfa & Ciclo biológico \\
\hline 18 & $7,7 \pm 0,67 \mathrm{a}$ & $35,8 \pm 0,71 \mathrm{a}$ & $43,5 \pm 1,31 \mathrm{a}$ \\
20 & $6,4 \pm 0,17 \mathrm{~b}$ & $24,5 \pm 0,34 \mathrm{~b}$ & $30,9 \pm 0,52 \mathrm{~b}$ \\
22 & $5,9 \pm 0,16 \mathrm{~b}$ & $23,8 \pm 0,36 \mathrm{~b}$ & $29,6 \pm 0,66 \mathrm{~b}$ \\
25 & $4,5 \pm 0,11 \mathrm{c}$ & $12,6 \pm 0,26 \mathrm{c}$ & $17,1 \pm 0,29 \mathrm{c}$ \\
28 & $3,2 \pm 0,19 \mathrm{~d}$ & $12,2 \pm 0,25 \mathrm{c}$ & $15,4 \pm 0,16 \mathrm{~cd}$ \\
30 & $2,9 \pm 0,17 \mathrm{~d}$ & $9,4 \pm 0,41 \mathrm{c}$ & $12,4 \pm 0,24 \mathrm{~d}$ \\
32 & $2,6 \pm 0,23 \mathrm{~d}$ & $9,4 \pm 0,66 \mathrm{c}$ & $12,1 \pm 0,37 \mathrm{~d}$ \\
\hline
\end{tabular}

Média seguidas da mesma letra, nas colunas, não diferem entre si, pelo teste de Tukey $(\mathrm{P} \leq 0,05)$.

Tabela 4. Viabilidade média ( \pm DPM) (dias) das fases de ovo e ninfa e do ciclo biológico (ovo-adulto) de Diaphorina citri criado em limão Cravo, em diferentes temperaturas. UR de $70 \pm 10 \%$ e fotofase de 14 horas

\begin{tabular}{cccc}
\hline \multirow{2}{*}{ Temperatura $\left({ }^{\circ} \mathrm{C}\right)$} & \multicolumn{3}{c}{ Duração (dias) } \\
\cline { 2 - 4 } & $95,0 \pm 0,68$ & Ninfa & Ciclo biológico \\
\hline 18 & $95,2 \pm 0,92$ & $70,0 \pm 2,04 \mathrm{a}$ & $69,9 \pm 1,31 \mathrm{a}$ \\
20 & $88,4 \pm 3,98$ & $72,5 \pm 2,72 \mathrm{a}$ & $66,6 \pm 1,94 \mathrm{a}$ \\
22 & $93,8 \pm 3,40$ & $74,0 \pm 4,64 \mathrm{a}$ & $64,1 \pm 2,63 \mathrm{a}$ \\
25 & $89,8 \pm 2,58$ & $77,5 \pm 3,98 \mathrm{a}$ & $69,5 \pm 2,98 \mathrm{a}$ \\
28 & $90,5 \pm 5,03$ & $73,8 \pm 3,65 \mathrm{a}$ & $66,8 \pm 0,83 \mathrm{a}$ \\
30 & $81,6 \pm 4,50$ & $15,0 \pm 2,77 \mathrm{~b}$ & $12,2 \pm 2,61 \mathrm{~b}$ \\
\hline 3
\end{tabular}

Médias seguidas da mesma letra, nas colunas, não diferem entre si, pelo teste de Tukey $(\mathrm{P} \leq 0,05)$

Tabela 5. Temperatura base (Tb), constante térmica $(\mathrm{K})$, equação da velocidade de desenvolvimento (1/D) e coeficiente de determinação $\left(\mathrm{R}^{2}\right)$ das fases do ciclo biológico de Diaphorina citri, criado em limão Cravo.

\begin{tabular}{lcccc}
\hline \multicolumn{1}{c}{ Fase / Período } & $\mathrm{Tb}\left({ }^{\circ} \mathrm{C}\right)$ & $\mathrm{K}(\mathrm{GD})$ & Equação $(1 / \mathrm{D})$ & $\mathrm{R}^{2}(\%)$ \\
\hline Ovo & 12,07 & 52,61 & $\mathrm{y}=0,019007 \mathrm{x}-0,229488$ & 0,9773 \\
Ninfa & 13,94 & 156,88 & $\mathrm{y}=0,006374 \mathrm{x}-0,088836$ & 0,9465 \\
Ovo-adulto & 13,53 & 210,91 & $\mathrm{y}=0,004741 \mathrm{x}-0,064134$ & 0,9670 \\
\hline
\end{tabular}

$2^{\mathrm{a}} \mathrm{e} 3^{\mathrm{a}}$ gerações, respectivamente (Paiva, 2009). Na $2^{\mathrm{a}}$ geração, também houve alta mortalidade de ninfas mais jovens. Tais constatações mostraram que essas etapas do desenvolvimento de $D$. citri são as mais vulneráveis, constituindo-se, assim, em fatores chaves do crescimento populacional deste inseto em campo, devendo ser o alvo de medidas de controle. A mortalidade de ninfas mais desenvolvidas foi semelhante nas três gerações estudadas, sendo 25,33 e $25 \%$ na $1^{\mathrm{a}}, 2^{\mathrm{a}}$ e $3^{\mathrm{a}}$ gerações, respectivamente (Paiva, 2009).

A densidade média de ovos de $D$. citri em condições de campo, no estado de São Paulo (8,7 ovos por ramo) (Paiva, 2009) é metade daquela verificada na Flórida, EUA (16,0 ovos por ramo, em média) (Hall et al., 2008). Curiosamente, no Brasil, o controle populacional por meio da atividade predatória é relativamente baixo se comparado ao verificado nos EUA (Michaud, 2004), o que pode estar associado à excessiva aplicação de produtos inseticidas para controle do vetor. Embora a viabilidade de ovo a adulto em campo tenha sido baixa nas gerações estudadas em São Paulo (1,7 a 21,4\%) (Paiva, 2009) e, portanto, inferior aos valores obtidos em laboratório (Nava et al., 2007), as exigências térmicas determinadas a partir 
de dados de laboratório (Liu \& Tsai, 2000; Nava et al., 2007) foram aplicáveis às condições de campo. A duração do período ovo-adulto foi muito semelhante nas gerações estudadas, com uma variação entre 18 e 24,7 dias (Paiva, 2009).

A umidade relativa do ar (UR) não influenciou a duração das fases de ovo e ninfa de $D$. citri, que permaneceram próximas de 5,5 e 16 dias, respectivamente. De forma análoga, a UR não afetou a viabilidade da fase de ovo, entretanto, a viabilidade ninfal foi reduzida para $40 \%$, sob UR de $30 \%$, diferindo significativamente das demais UR (Figura 4).

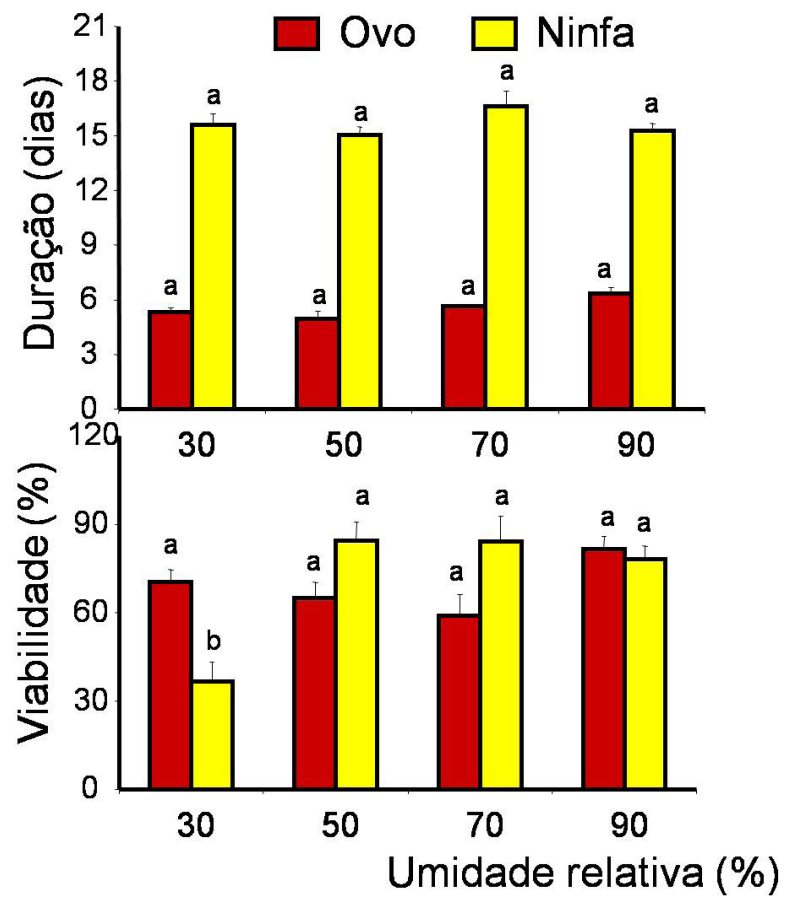

Figura 4. Efeito da umidade relativa do ar sobre a duração e a viabilidade das fases de ovo e ninfa de Diaphorina citri. Temperatura de $25 \pm 2^{\circ} \mathrm{C}$ e fotofase de 14 horas (Nava et al., 2007).

\section{DINÂMICA POPULACIONAL DE D. citri}

Não há um padrão de distribuição temporal de $D$. citri para o estado de São Paulo, sendo tal distribuição variável em função da disponibilidade e abundância de ramos novos, embora os fatores climáticos também sejam responsáveis pelo tamanho de suas populações (Paiva, 2009). As maiores infestações de ninfas de $D$. citri, considerando as proporções de ramos infestados por essa fase do inseto, foram observadas em pomares de São Carlos e as menores, em Araras. As maiores infestações de adultos foram constatadas em Bauru, Botucatu, Lins e São Carlos e as menores, em Araras e Casa Branca. É conveniente salientar que, independente da região, o psilídeo apresenta um padrão de distribuição espacial do tipo agregada (Paiva, 2009).

\section{ALTERNATIVAS DE CONTROLE DO PSILÍDEO D. citri}

Dentre as várias alternativas de controle do vetor, além do químico, existem os métodos biológicos (parasitoides, predadores e patógenos) e comportamentais.

Embora os predadores possam ser importantes em outros países (Michaud, 2004), não têm se mostrado eficientes no Brasil (Paiva, 2009), talvez, conforme já comentado, devido à aplicação sistemática e, muitas vezes, irracional de produtos químicos.

Dentre os parasitoides da espécie ocorrente no Brasil, optou-se pelo ectoparasitóide Tamarixia radiata (Waterston) (Hymenoptera: Eulophidae) em relação ao endoparasitoide Diaphorencyrtus aligarhensis (Shafee, Alam \& Agarwal) (Hymenoptera: Encyrtidae), pelos registros de sucesso da primeira espécie nas Ilhas Reunión em 1978 e em Guadalupe em 1999, com liberações de 4.600 e de 1000 adultos, em ambos os locais, respectivamente (Étiene et al, 2001). Inicialmente, pensou-se na importação do parasitoide, originário da Ásia, mas que vinha sendo criado na Flórida, EUA, e foram efetuados contatos com a Dra. Marjorie Hoy da Universidade da Flórida, Gainesville, para início do processo de importação. Entretanto, nos anos de 2005-2006, nos municípios de Jaboticabal e Piracicaba, SP, encontrou-se a referida espécie (Gómez Torres et al., 2006), o que facilitou o início dos estudos de controle biológico dessa praga no Brasil (Figura 2).

Foram coletados espécimens de $T$. radiata em diferentes regiões do estado de São Paulo e, a despeito da grande variação policromática, testes moleculares usando marcadores revelaram alta similaridade entre tais variações (Gómez Torres, 2009). O parasitismo observado foi bastante alto em diferentes regiões do estado de São Paulo (Figura 5).

Foram realizados estudos da biologia de $T$. radiata em diferentes temperaturas e elaborada a tabela de vida de fertilidade, concluindo-se que a temperatura 


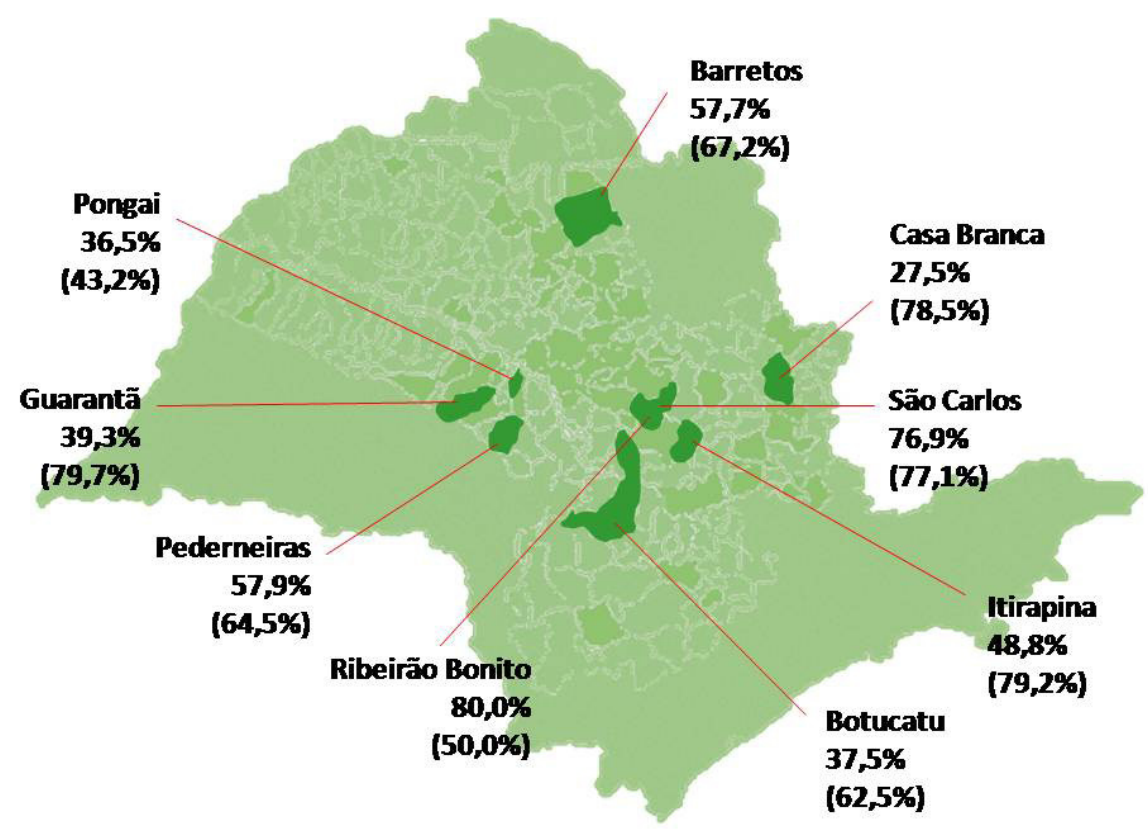

Figura 5. Taxa de parasitismo e de emergência (entre parênteses) de Tamarixia radiata em Diaphorina citri, em diferentes regiões do estado de São Paulo (Gómez Torres et al., dados não publicados).

de $25^{\circ} \mathrm{C}$ é a mais adequada ao desenvolvimento do inseto, por acarretar uma taxa líquida de reprodução (Ro) de 126,79, ou seja, uma capacidade de aumento de 127 vezes a cada geração, valor superior ao obtido nas demais temperaturas (Tabelas 6 e 7). A ação do parasitoide foi maior entre 25 e $30^{\circ} \mathrm{C}$, com maior emergência na faixa de 25 a $35^{\circ} \mathrm{C}$.

Maior porcentual de parasitismo foi verificado quando adultos de $T$. radiata se alimentaram de mel e pólen, havendo acentuada preferência por ninfas de $4^{\circ} \mathrm{e}$ $5^{\circ}$ instares de $D$. citri. A capacidade total de parasitismo

Tabela 6. Duração média de Tamarixia radiata criado em Diaphorina citri em diferentes temperaturas. UR de $70 \%$ e fotofase de $14 \mathrm{~h}$

\begin{tabular}{cc}
\hline Temperatura $\left({ }^{\circ} \mathrm{C}\right)$ & Duração (dias) \\
\hline 18 & $17,31 \mathrm{a}$ \\
20 & $14,20 \mathrm{~b}$ \\
22 & $12,46 \mathrm{c}$ \\
25 & $10,33 \mathrm{~d}$ \\
28 & $10,09 \mathrm{~d}$ \\
30 & $7,55 \mathrm{e}$ \\
32 & $7,59 \mathrm{e}$
\end{tabular}

Teste de Tukey ( $\mathrm{P}>0,05)$ (Gómez Torres et al., dados não publicados em preparação). foi maior a $25^{\circ} \mathrm{C}$ (167 ninfas parasitadas) (Figura 6), embora o total de ninfas parasitadas tenha sido bem inferior ao referido na literatura (Chu \& Chien, 1991; Skelley \& Hoy, 2004), com registros de 500 ninfas destruídas pelo parasitoide.

Com relação à planta hospedeira, houve uma variação na taxa de parasitismo em função da variedade de citros infestada, observando-se maior porcentual na variedade Hamlin (35,6\%), seguida pela Pêra (19,2\%), Valência $(8,6 \%)$ e Natal (7,3\%) (Paiva, 2009).

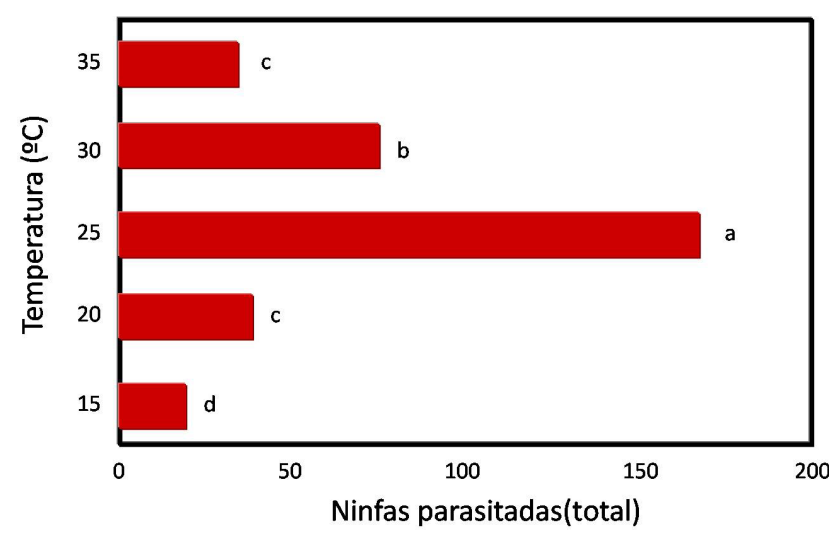

Figura 6. Capacidade de parasitismo de T. radiata em diferentes temperaturas (Gómez Torres et al., dados não publicados). 
Tabela 7. Tabela de vida de fertilidade de Tamarixia radiata criado em Diaphorina citri em diferentes temperaturas. UR de $70 \%$ e fotofase de $14 \mathrm{~h}$

\begin{tabular}{crcc}
\hline Temperatura $\left({ }^{\circ} \mathrm{C}\right)$ & \multicolumn{1}{c}{ Ro } & $\mathrm{r}_{\mathrm{m}}$ & \multicolumn{1}{c}{$\lambda$} \\
\hline 15 & 9,88 & 0,1826 & 1,2003 \\
20 & 23,62 & 0,2531 & 1,2880 \\
25 & 126,79 & 0,3742 & 1,4538 \\
30 & 58,63 & 0,3378 & 1,4019 \\
35 & 21,27 & 0,2528 & 1,2889 \\
\hline
\end{tabular}

(Gómez Torres et al., dados não publicados).

Em condições de laboratório, tem-se observado um aumento na taxa de parasitismo nas populações de T. radiata que apresentam a bactéria Wolbachia, com uma variação de 30,3 para $19,8 \%$ de parasitismo em indivíduos com o simbionte em relação àqueles sem a bactéria (Gómez Torres et al., em preparação).

Em liberações inoculativas de $T$. radiata feitas em campo, observou-se, após oito dias, que a porcentagem de parasitismo se manteve entre 51,6 e $72,8 \%$, valores que decresceram para 4,2 e $10 \%$ após 15 dias (Gómez Torres, 2009). Em cultivos nos municípios de Cordeirópolis e Limeira, SP, após três liberações a porcentagem de parasitismo de $T$. radiata variou entre 30 e $60 \%$, constatando-se que o número de ninfas de $4^{\circ}$ e $5^{\circ}$ ínstares decresceu nas áreas onde foram liberados.

Com base nas exigências térmicas e higrométricas de $D$. citri e de $T$. radiata (Tabela 8) elaborou-se um zoneamento em um mapa de isotermas (Figura 7), por meio do qual verificou-se que, para cada geração de $D$. citri, existem de 2,6 a 3,$1 ; 3,1$ a 4,0; 3,1 a 6,3 e 3,1 a 6,3 gerações de $T$. radiata, respectivamente, para as regiões Norte, Nordeste, Sudoeste e Sudeste do estado de São Paulo (Gómez Torres, 2009).

$\mathrm{O}$ parasitoide $T$. radiata vem sendo estudado em diversos países, procurando-se melhorar a tecnologia de produção em larga escala. Em recente Workshop realizado em McAllen, Texas, em fevereiro de 2010, discutiram-se diretrizes para produção do psilídeo e do seu parasitoide. No Brasil, a técnica de criação de $T$. radiata vem sendo muito semelhante àquela utilizada para produção de Ageniaspis citricola (Logvinovskaya) (Hymenoptera, Encyrtidae) para controle de Phyllocnistis citrella Stainton (Lepidoptera: Gracillariidae) em citros (Chagas et al., 2002) (Figura 8).

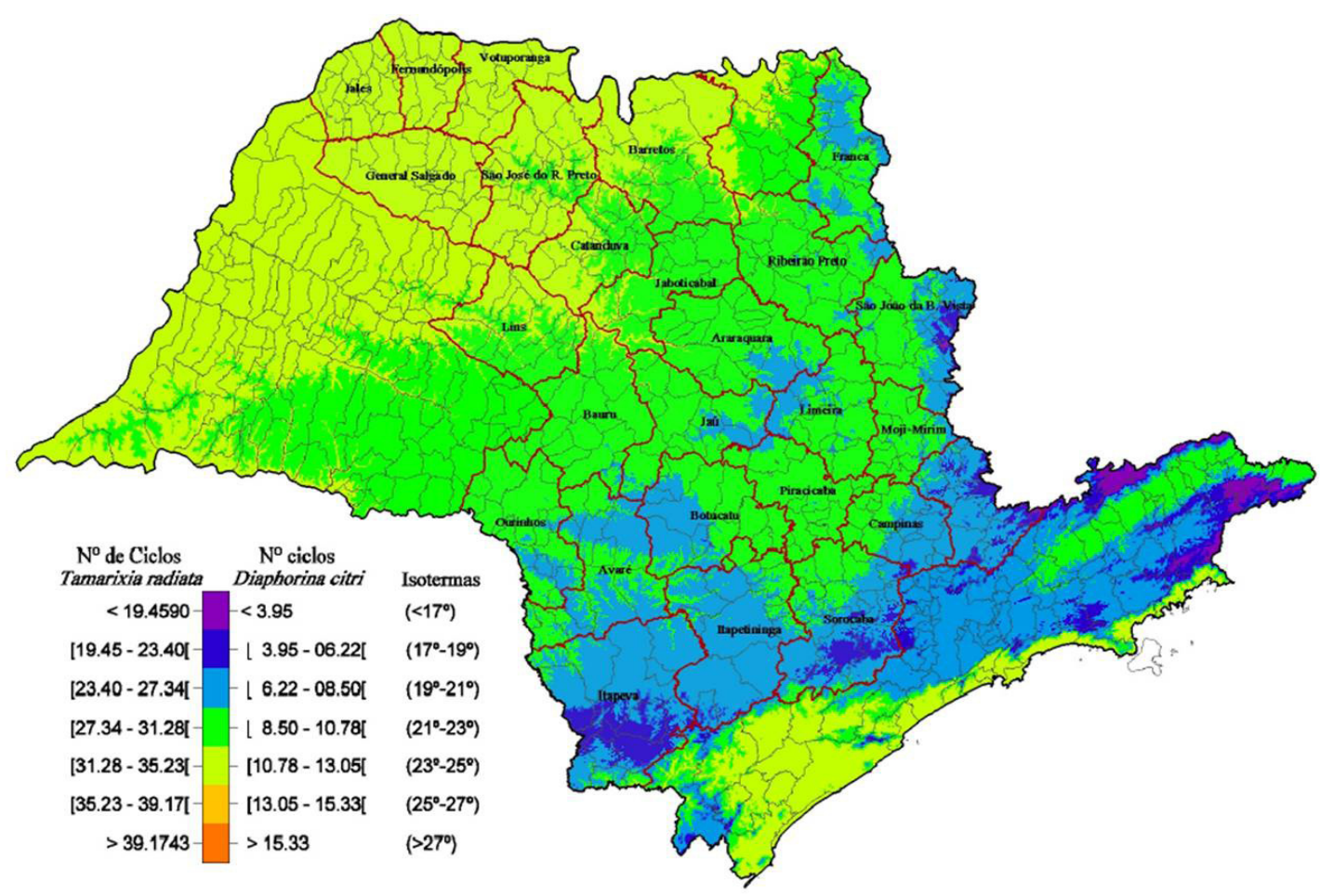

Figura 7. Número de ciclos anuais de Diaphorina citri e Tamarixia radiata em São Paulo, com base em um mapa de isotermas (Gómez Torres et al., dados não publicados). 
Tabela 8. Exigências térmicas e faixa de umidade favorável a Diaphorina citri e a Tamarixia radiata

\begin{tabular}{lcc}
\hline Temperatura $\left({ }^{\circ} \mathrm{C}\right)$ & D. citri & T. radiata \\
\hline $\begin{array}{l}\text { Tb (limiar térmica inferior } \\
\text { de desenvolvimento) }\end{array}$ & $13,5^{\circ} \mathrm{C}$ & $7,1^{\circ} \mathrm{C}$ \\
$\begin{array}{l}\text { K (constante térmica em } \\
\text { graus dias) }\end{array}$ & $210,9 \mathrm{GD}$ & $187,5 \mathrm{GD}$ \\
UR faixa favorável & $70-85 \%$ & $70 \%$ \\
\hline
\end{tabular}

A utilização do parasitoide como agente de controle biológico do psilídeo poderá ser um componente adicional ao manejo integrado de pragas (MIP) em citros e, para tal, é necessário que se melhore a técnica de criação do inimigo natural. Entretanto, a maior restrição ao uso do parasitoide é a aplicação sistemática de produtos químicos não seletivos para controlar o vetor. $\mathrm{O}$ parasitoide adulto e o seu parasitismo são muito afetados pelos produtos químicos atualmente utilizados na citricultura (Carvalho, 2008; Ferrari et al., 2010).

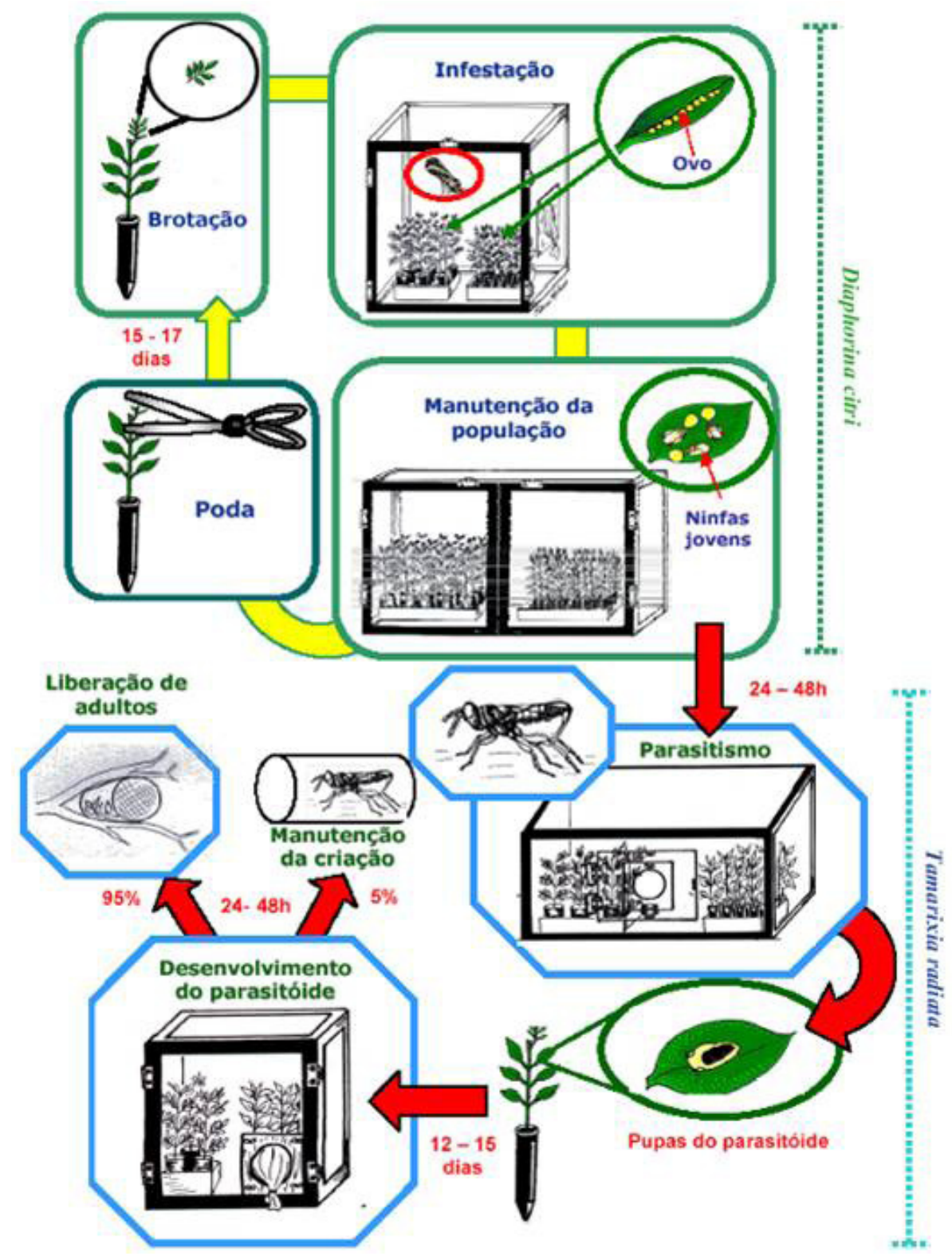

Figura 8. Esquema de produção de Tamarixia radiata (Gómez Torres et al., dados não publicados). 
A utilização de $T$. radiata poderá ser feita em áreas orgânicas, ou mesmo em áreas de grande ocupação pelo hospedeiro alternativo do psilídeo, a planta ornamental conhecida como murta-de-cheiro, M. paniculata, visando incrementar a população do parasitoide no campo. Há necessidade de validar o modelo de zoneamento no campo e outros já desenvolvidos (Hamada et al., 2007), para que se possa selecionar e liberar populações do parasitoide nas áreas problemas, bem como, incrementar sua produção no sistema atualmente utilizado.

Patógenos como Beauveria bassiana (Padulla \& Alves, 2009) ou mesmo voláteis da goiaba, Psidium guajava, que atuam como repelentes do psilídeo (em fase de estudos no mundo) poderão ser incluídos futuramente como componentes do MIP da cultura cítrica.

\section{TRANSMISSÃO DE Candidatus Liberibacter spp.}

Embora possa causar danos à planta cítrica pela sucção de seiva e deformação foliar em brotos jovens quando em altas populações (Halbert \& Manjunath, 2004; Michaud, 2004), D. citri tornou-se importante pela transmissão de bactérias fitopatogênicas do gênero Candidatus Liberibacter, associadas ao HLB. Assim, a transmissão dessas bactérias pelo psilídeo é um processo biológico que deve ser compreendido, principalmente as características de importância epidemiológica, tais como períodos de tempo necessários para aquisição e inoculação, latência, persistência e eficiência de transmissão.

A transmissão por psilídeos foi demonstrada pela primeira vez para o HLB africano, usando-se Trioza erytreae como vetor (McLean \& Oberholzer, 1965). Em seguida, verificou-se a transmissão do HLB asiático por um outro psilídeo vetor nas Filipinas (Salibe \& Cortez, 1966), identificado como D. citri (Martinez \& Wallace, 1967). Doenças anteriormente designadas como HLB africano (ou greening) e HLB asiático estão associadas às bactérias $C a$. L. africanus e $C a$. L. asiaticus, respectivamente (Bové, 2006). A nova espécie $\mathrm{Ca}$. L. americanus, descrita recentemente em citros no Brasil (Teixeira et al., 2005), é transmitida por D. citri (Yamamoto et al., 2006).
O processo de transmissão de bactérias fitopatogênicas por insetos inicia-se com a aquisição do patógeno em uma planta infectada e termina com a inoculação do mesmo em uma planta sadia. Entre as etapas de aquisição e inoculação, as células bacterianas podem ficar restritas a um sítio de retenção específico, como a porção anterior do tubo digestivo (estomodeu) do vetor, no caso da transmissão de Xylella fastidiosa por cigarrinhas (Lopes, 1996), ou circular e se multiplicar em vários tecidos do corpo do inseto vetor, incluindo glândulas salivares, como no caso de fitoplasmas (Weintraub \& Beanland, 2006). No segundo caso, há um período latente de vários dias ou semanas entre o início da aquisição e a inoculação, que ocorre via saliva, após a colonização das glândulas salivares. O mecanismo de transmissão de $C a$. Liberibacter por psilídeos ainda não está totalmente esclarecido, mas pelas informações disponíveis até o momento parece seguir o modelo de circulação e/ou propagação em vários tecidos, culminando com inoculação via saliva, semelhante ao de fitoplasmas.

A aquisição de $C a$. L. asiaticus pode ocorrer tanto por ninfas $\left(4^{\circ}\right.$. e $5^{\circ}$. ínstares) como por adultos de D. citri (Xu et al., 1988), porém as ninfas adquirem com maior eficiência (Vichin Neto et al., 2008). Indivíduos que adquirem o patógeno na fase ninfal são capazes de transmitir após a ecdise para a fase adulta (Inoue et al., 2009), evidenciando que o patógeno é circulativo no vetor, não estando restrito ao estomodeu ou às peças bucais. Após a aquisição do patógeno em plantas infectadas, é necessário um tempo para que $D$. citri seja capaz de inocular a bactéria em uma planta sadia. Esta característica, conhecida como latência, tem sido relatada para a transmissão das formas asiática e africana de HLB. Entretanto, os períodos de latência relatados são muito contrastantes, variando de $24 \mathrm{~h}$ (Buitendag \& von Broembsen, 1993), 8-12 dias (Graça, 1991) a até 21 dias (Capoor et al., 1974). Por meio da técnica de PCR quantitativo, demonstrou-se um aumento na concentração de $C a$. L. asiaticus em $D$. citri com o passar do tempo após a aquisição pelas ninfas (Vichin Neto et al., 2008; Inoue et al., 2009), indicando que a transmissão é do tipo propagativa. A ocorrência de multiplicação da bactéria no vetor também é evidenciada pela longa persistência da transmissão. Hung et al. (2004) mostraram que adultos de D. citri 
permanecem infectivos com $\mathrm{Ca}$. L. asiaticus por até 12 semanas após a aquisição em plantas infectadas. Para $C a$. L. americanus e $C a$. L. asiaticus, observou-se transmissão para plantas cítricas após várias semanas da aquisição (J.R.S. Lopes, dados não publicados), o que caracteriza a transmissão como persistente.

Estudos mais aprofundados da relação entre $C a$. Liberibacter spp. e D. citri são necessários para identificar as rotas e mecanismos de circulação e multiplicação do patógeno no inseto vetor. O patógeno já foi observado na hemolinfa e glândula salivar do vetor (Moll \& Martin 1973; Xu et al. 1988), porém ainda não há evidências diretas de sua inoculação via saliva. A transmissão vertical do patógeno para a progênie através dos ovos (transmissão transovariana) não foi constatada para D. citri (Capoor et al., 1974, Hung et al., 2004), mas esta possibilidade não deve ser descartada, já que para $T$. erytreae há evidência deste tipo de transmissão (Van den Berg et al., 1991-92).

Ainda não há informações sobre a eficiência de transmissão de $C a$. Liberibacter spp. por D. citri no Brasil. Estudos realizados na Ásia registraram taxas de transmissão muito variáveis (1-100\%) para a forma asiática de HLB (Capoor et al., 1974; Huang et al., 1984; Xu et al., 1988), possivelmente devido a diferenças em biótipos do psilídeo (Su, 2001), estirpes da bactéria ou condições experimentais nos diferentes trabalhos, tais como temperatura e períodos de acesso à aquisição ou inoculação. Experimentos variando-se o período de acesso à aquisição em plantas infectadas, ou o período de acesso à inoculação em plantas sadias, indicaram que adultos de $D$. citri podem adquirir ou inocular a forma asiática deste patógeno em um tempo mínimo de 15-30 min (Capoor et al. 1974) ou 5 h (Xu et al. 1988). Para o HLB africano, a inoculação pode ocorrer em menos de $1 \mathrm{~h}$ (van Vuuren \& Graça 1977). Entretanto, períodos mais longos de acesso à aquisição e inoculação devem aumentar a eficiência de transmissão desses patógenos. Conforme mencionado anteriormente, o estágio de desenvolvimento do inseto em que ocorre a aquisição também pode influenciar os resultados, já que após aquisição por ninfas o patógeno atinge maiores concentrações no inseto vetor do que quando a aquisição ocorre na fase adulta (Inoue et al., 2009).
Em geral, as interações patógeno-vetor são pouco estudadas para as diversas formas de HLB, sendo que a maioria dos estudos foi realizada na África do Sul e na Ásia. Novos estudos enfocando a transmissão de $C a$. Liberibacter asiaticus e $C a$. L. americanus por $D$. citri foram iniciados após a detecção destes patógenos no Brasil (2004) e nos EUA (2005), mas ainda há poucas informações publicadas. A recente descoberta de uma nova espécie de $\mathrm{Ca}$. Liberibacter associada a doenças em batata ('Zebra chip'), tomateiro e outras solanáceas, denominada $\mathrm{Ca}$. L. solanacearum (Liefting et al. 2008) e transmitida pelo psilídeo Bactericera cockerelli (Sulc) (Hansen et al., 2008), deve impulsionar ainda mais os estudos de transmissão deste grupo de patógenos, esclarecendo suas relações com os psilídeos vetores.

\section{AGRADECIMENTOS}

À Fapesp, pelo financiamento do projeto temático sobre o assunto, ao Fundecitrus pelo financiamento de parte das pesquisas e ao CNPq, pela bolsa de produtividade ao primeiro, segundo e quarto autores. Agradecemos também ao CNPq, Fapesp e MCT pelo financiamento do INCT- "Semioquímicos na Agricultura" e no qual o vetor $D$. citri vem sendo estudado.

\section{REFERÊNCIAS BIBLIOGRÁFICAS}

Bassanezi RB, Montesino LH, Busato LA \& Stuchi ES (2006) Damages caused by Huanglongbing on sweet orange yield and quality in São Paulo. Proceedings of the Huanglongbing - Greening International Workshop. Araraquara, SP: Fundecitrus. Resumo S17.

Bové JM (2006) Huanglongbing: a destructive, newly emerging, century-old disease of citrus. Journal of Plant Pathology 88: 7-37.

Buitendag CH \& von Broembsen LA(1993) Living with citrus greening in South Africa. In: Moreno P, Graça JV \& Timmer LW (Eds.) Proceedings of the Twelfth Conference of the International Organization of Citrus Virologists. University of California, Department of Plant Pathology, Riverside, CA, p. 269-273.

Capoor SP, Rao DG \& Viswanath SM (1974) Greening disease of citrus in the Deccan Trap Contry 
and its relationship with the vector, Diaphorina citri Kuwayama. In: Weathers LG \& Cohen M (Eds.) Proceedings of the $6^{\text {th }}$ Conference of the International Organization of Citrus Virologists, University of California, Division of Agricultural Sciences, p.43-49.

Carvalho SPL (2008) Toxicidade de inseticidas neonicotinóides sobre o psilídeo Diaphorina citri Kuwayama (Hemiptera: Psyllidae) e o parasitóide Tamarixia radiata (Waterston) (Hymenoptera: Eulophidae). Tese de doutorado - Escola Superior de Agricultura "Luiz de Queiroz", Universidade de São Paulo, Piracicaba. 59p.

Chagas MCM, Parra JRP, Milano P, Nascimento AM \& Parra ALGC (2002) Ageniaspis citricola: criação e estabelecimento no Brasil. In: Parra, JRP, Botelho PSM, Corrêa-Ferreira BS \& Bento JMS (Eds). Controle Biológico no Brasil: parasitóides e predadores, p.377-394.

Chu YI \& Chien CC (1991) Utilization of natural enemies to control psyllid vectors transmitting citrus greening. In: Kiritani K, Su HJ \& Chu YI (Eds.) Integrated control of plant virus diseases. Taipei: Food and Fertilizer Technology Center for the Asian and Pacific Region, p.135-145.

Coletta-Filho HD, Targon MLPN, Takita MA, De Negri JD, Pompeu Jr J, Machado MA, Do Amaral AM \& Muller GW (2004) First Report of the Causal Agent of Huanglongbing ("Candidatus Liberibacter asiaticus") in Brazil. Plant Disease 88:1382.

Costa Lima AM (1942) Insetos do Brasil, Homoptera. Rio de Janeiro: Escola Nacional de Agronomia 3: 101.

Étienne J, Quilici S, Marival D \& Franck A (2001) Biological control of Diaphorina citri (Hemiptera: Psyllidae) in Guadeloupe by imported Tamarixia radiata (Hymenoptera: Eulophidae). Fruits 56: 307-315.

Ferrari BM, Omoto C, Rochelle ATFA \& Sumiya BKI (2010) Efeito letal e subletal de inseticidas sobre Tamarixia radiata (Waterston, 1922) (Hymenoptera: Eulophidae). PAB (no prelo).

Gallo D, Nakano O, Silveira Neto S, Carvalho RPL, Baptista GC, Berti Filho E, Parra JRP, Zucchi RA, Alves SB, Vendramim JD, Marchini LC, Lopes JRS \& Omoto C (2002) Entomologia Agrícola. Piracicaba: FEALQ, $920 \mathrm{p}$.
Gómez Torres ML (2009) Estudos biológicos de Tamarixia radiata (Waterston, 1922) (Hymenoptera: Eulophidae) para o controle de Diaphorina citri Kuwayama, 1907 (Hemiptera: Psyllidae). Tese de doutorado - Escola Superior de Agricultura "Luiz de Queiroz”, Universidade de São Paulo, Piracicaba. 138p.

Gómez Torres ML, Nava DE, Gravena S, Costa VA \& Parra JRP (2006) Registro de Tamarixia radiata (Waterston) (Hymenoptera: Eulophidae) em Diaphorina citri (Hemiptera: Psyllidae) em São Paulo, Brasil. Revista de Agricultura 81:112-117.

Graça JV (1991) Citrus greening disease. Annual Review of Phytopathology 29:109-136.

Halbert SE \& Manjunath KL (2004) Asian citrus psyllids (Sternorrhyncha: Psyllidae) and greening disease of citrus: a literature review and assessment of risk in Florida. Florida Entomologist 87:330-353.

Halbert SE \& Nuñez CA (2004) Distribution of the Asian citrus psyllid, Diaphorina citri Kuwayama (Rhynchota: Psyllidae) in the Caribbean basin. Florida Entomologist 83:401-402.

Hall DG, Hentz MG \& Adair JRRC (2008) Population ecology and phenology of Diaphorina citri (Hemiptera: Psyllidae) in two Florida citrus groves. Environmental Entomology 37: 914-924.

Hamada E, Morandi MAB, Tambasco FJ, Pereira DA \& Evangelista $S$ (2007) Estimativa de áreas favoráveis à ocorrência de Diaphorina citri (vetor do greening asiático) no Estado de São Paulo. Jaguariúna: Embrapa Meio Ambiente. Disponível em: http://www.cenargen.embrapa. br/publica/trabalhos/cot058.pdf. Acesso em 10 jun. 2008.

Hansen AK, Trumble JT, Stourhamer R \& Paine TD (2008) A new huanglongbing species, "Candidatus Liberibacter psyllaurous," found to infect tomato and potato, is vectored by the psyllid Bactericera cockerelli (Sulc). Applied Environmental Microbiology 74:58625865.

Hoy MA \& Nguyen R (2001) Classical biological control of Asian citrus psylla. Citrus Industry 81: 48-50.

Huang CH, Tsai MY \& Wang CL (1984) Transmission of citrus likubin by a psyllid, Diaphorina citri. Journal of Agricultural Research of China 33: 15-72. 
Hung TH, Hung SC, Chen CN, Hsu MH \& Su HJ (2004) Detection by PCR of Candidatus Liberibacter asiaticus, the bacterium causing citrus huanglongbing in vector psyllids: application to the study of vectorpathogen relationships. Plant Pathology 53:96-102.

Inoue $\mathrm{H}$, Ohnish J, Ito $\mathrm{T}$, Tomimura $\mathrm{K}$, Miyata $\mathrm{S}$, Iwanami T \& Ashihara W (2009) Enhanced proliferation and efficient transmission of Candidatus Liberibacter asiaticus by adult Diaphorina citri after acquisition feeding in the nymphal stage. Annals of Applied Biology 155:29-36.

Liefting LW, Weir BS, Pennycook SR \& Clover GRG (2008) 'Candidatus Liberibacter solanacearum', a liberibacter associated with plants in the family Solanaceae. International Journal of Systematic and Evolutionary Microbiology 59:2274-2276.

Liu, YH \& Tsai, JH (2000) Effects of temperature on biology and life table parameters of the Asian citrus psyllid, Diaphorina citri Kuwayama (Homoptera: Psyllidae). Annals of Applied Biology 137:201-206.

Lopes JRS (1996) Mecanismos de transmissão de Xylella fastidiosa por cigarrinhas. Laranja 17:79-92.

Martinez AL \& Wallace JM (1967) Citrus leaf mottleyellows disease in the Philippines and transmission of the causal virus by a psyllid, Diaphorina citri. Plant Disease Reporter 51:692-695.

McLean ADP \& Oberhoizer PCT (1965) Citrus psylla, a vector of the greening disease of sweet orange. South African Journal of Science 8:297-298.

Michaud JP (2004) Natural mortality of Asian citrus psyllid (Homoptera: Psyllidae) in central Florida. Biological Control 29: 260-269.

Moll JN \& Martin MN (1973) Electron microscope evidence that citrus psylla (Trioza erytreae) is a vector of greening disease in South Africa. Phytophylactica 5:41-44.

Nava DE, Torres MLG, Rodrigues MDL, Bento JMS \& Parra JRP (2007) Biology of Diaphorina citri (Hem., Psyllidae) on different hosts and at different temperatures. Journal of Applied Entomology 131: 709-715.

Padulla LFL \& Alves SB (2009) Suscetibilidade de ninfas de Diaphorina citri a fungos entomopatogênicos. Arquivos do Instituto Biológico 76: 297-902.
Paiva PEB (2009) Distribuição espacial e temporal, inimigos naturais e tabela de vida ecológica de Diaphorina citri Kuwayama (Hemiptera: Psyllidae) em citros em São Paulo. Tese de doutorado-Escola Superior de Agricultura "Luiz de Queiroz", Universidade de São Paulo, Piracicaba. 64p.

Salibe AA \& Cortez RE (1966) Studies on the leaf mottling disease of citrus in the Philippines. FAO Plant Prot. Bull. 14:141-144.

Skelley LH \& Hoy MA (2004) A synchronous rearing method for the Asian citrus psyllid and its parasitoids in quarantine. Biological Control 29:14-23.

$\mathrm{Su}$ HJ (2001) Citrus greening disease. Food and Fertilizer Technology Center Technical notes. Plant Protection, $\mathrm{N}^{\mathrm{o}}$ 2001-2.

Teixeira DC, Ayres AJ, Kitajima EW, Tanaka FAO, Danet L, Jagoueix-Eveillard S, Saillard C \& Bové JM (2005) First Report of a Huanglongbing-Like Disease of Citrus in Sao Paulo State, Brazil and Association of a New Liberibacter Species, "Candidatus Liberibacter americanus", with the Disease. Plant Disease 89: 107.

Tsai JH, Wang JJ \& Liu YH (2002) Seasonal abundance of the Asian citrus psyllid, Diaphorina citri (Homoptera: Psyllidae) in southern Florida. Florida Entomologist 85: 446-451.

van den Berg MA, van Vuuren SP \& Deacon VE (199192) Studies on greening disease transmission by the citrus psylla, Trioza erytreae (Hemiptera: Triozidae). Israel Journal of Entomology 25-26:51-56.

van Vuuren SP \& Graça JV (1977) The effects of exposure time and monocrotophos on citrus greening transmission by psylla (Trioza erytreae). Citrus Subtropical Fruit Journal 536:13-14.

Vichin Neto R, Nascimento FE, Balbinotte J, Coletta-Filho H \& Lopes JRS (2008) Comparação da capacidade de aquisição de Candidatus Liberibacter asiaticus em citros por ninfas e adultos de Diaphorina citri Kuwayama (Hemiptera:Psyllidae). Resumos do $16^{\circ}$ Simpósio Internacional de Iniciação Científica USP. São Paulo, SP.

Weintraub PG \& Benland L (2006) Insect vectors of phytoplasmas. Annual Review of Entomology 51: 91-111. 
Xu CF, Xia YH, Li KB \& Ke C (1988) Further study of the transmission of citrus huanglongbing by a psyllid, Diaphorina citri Kuwayama. In: Timmer LW, Garnsey SM \& Navarro L (Eds). Proceedings of the 10th Conference of the International Organization of Citrus Virologists. University of California, Riverside. p.243248.

Yamamoto PT, Felippe MR, Garbim, LF, Coelho, JHC, Ximenes NL, Martins EC, Leite APR, Souza MC, Abraão DP \& Braz JD (2006) Diaphorina citri (Kuwayama) (Hemiptera: Psyllidae): vector of the bacterium Candidatus Liberibacter americanus. In: Proceedings of the Huanglongbing-Greening International Workshop. Araraquara, SP: Fundecitrus, p.96.

Yamamoto PT, Paiva PEB \& Gravena S (2001) Flutuação Populacional de Diaphorina citri Kuwayama (Hemiptera: Psyllidae) em Pomares de Citros na Região Norte do Estado de São Paulo. Neotropical Entomology 30:165-170. 